\title{
Alternative approach to international comparisons
}

\author{
Samoilenko Oleksandr ${ }^{1}$ \\ ${ }^{1} \mathrm{SE} \ll$ Ukrmetrteststandard» \\ E-mail:asam@ukrcsm.kiev.ua
}

\begin{abstract}
According to the authors point of view, absence of the accepted by metrological society software based on simple and understandable and at the same time flexible and multipurpose mathematical apparatus is one of the restrictions on the way to arrangement of international comprehensive comparisons with expanded group of participants (hereinafter - comprehensive comparisons). General methodology of measurements adjustment by the least square method (LSM) may be used for this mathematical apparatus.

Software named «Metrology Network» is defined. It was used for repeated processing of measurement results during the key comparisons of EURAMET.L-K1.2011 «Measurement of gage blocks by interferometer». «Metrology Network» software allowed to estimate simply and quickly systematic measurement error components of the gage blocks length measurement by each laboratory. They are called multiplicative and additive measurement standards degrees of equivalence. «Metrology Network» allowed to estimate strictly by LSM the uncertainties of length measurement. Additive degrees of equivalence are considered substantial and constant characteristics of the measurement standards for many laboratories. Thus, they coincide for steel and ceramic gage blocks. These additive degrees of equivalence may be used as corrections in the process of calibration or as the reference values for further comparisons.

The simulation of measurement results for international comprehensive comparisons with complicated structure was provided. For 120 laboratories divided into 12 groups, totally 2442 length measurements of the steel or ceramic gage blocks were provided. They form together 24 sets of 8 gage blocks and provide 24 loops of comparisons. Necessity to provide the adjustment by the least square method is caused by the fact that two subgroups from each group of three laboratories took part in two different related loops of comparison. As a conclusion, «Metrology Network» software easily managed this complicated task of adjustment. It allowed us to calculate each of 120 measurement standards additive degree of equivalence relative to the averaged zero for all measurement standards and multiplicative degree of equivalence relative to the averaged measurement unit.
\end{abstract}

10.09.21

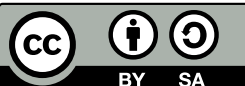

Keywords: international comprehensive comparison, gage blocks, adjustment, least square method, key comparisons reference value, additive and multiplicative degrees of equivalence, parameters, measurement standard, uncertainty.

\section{Foreword}

One of the metrology as a science tasks is improvement of the measurement results processing methods for receiving of more complete information concerning the structure of measurements biases for analysis. One of the modern requirements to such methods is their easy algorithmization for development of new high-performance software for processing of the measured data bulk with complicated structure. The purpose of this publication is to start discussion about all aspects of such software development and multipurpose clear mathematical basis provided for it.

\section{Actuality and purpose of the publication}

Key comparisons of the measurement standards are the highest rank of the traceability chain by the concept. The main purpose for next calibrations of 
the lower rank measurement standards according to the traceability chain is estimation of the systematic measurement error component relative to the higher rank measurement standards. Estimation of this systematic component is called bias. In the process of each measurement standard comparison the degree of equivalence is estimated in each measurement point, which is actually bias in this measurement point. This bias (degree of equivalence) may be used as correction in the process of calibration of the lower rank measurement standard in particular measurement point.

In the process of biases (degrees of equivalence) use arises a number of problems. Let's briefly discuss two of them. First of them is that comparisons are provided in particular discrete points of the measurement range. Number of these points may be smaller than necessary for calibration. Thus, measurement in the calibration process of the lower rank measurement standards on this particular measurement standard should be provided in other points, including random points of the measurement range. The second one is that reliability of biases (degrees of equivalence) is insufficient in each particular measurement point, in which comparisons were provided. Of course, the reliability measure is the uncertainty of measurements by the standard. This uncertainty in particular measurement point estimated by the laboratory may be insufficient for necessary reliability of using bias as a correction.

Widely spread method to cope with this problem is the approximation of biases in measurement points using a particular function, by the least square method (LSM). Any continuous function may be used but the simplest and widely spread is the linear one. Correction is easily calculated in each point of measurement range according to the parameters of function received from approximation. Likewise, uncertainty of correction calculation is easily estimated using statistical and other data received from the approximation. The defined above is easily realized for all units of the metrological traceability chain, except the first one, which is key comparisons. However, in the key comparisons process the measurement results of the measurement standards can be compared only with each other. Based on the facts, biases (degrees of equivalence) were estimated only in the measurement points recently as defined in the article ${ }^{[1]}$. Biases (degrees of equivalence) were estimated as the differences between the results of the laboratory and average or weighted average value in this point. These simple calculations are also a partial case of LSM.

It was offered for the first time to consolidate mentioned simple and certain procedures of the approximation and averaging to the entire one, which was realized in the publications ${ }^{[2,3]}$. This procedure realizes the general methodology of measurement results adjustments by the least square method. However, the key comparisons reference values (KCRV) are included to the offered measurement model together with measurement standards additive and multiplicative degrees of equivalence, which are coefficients of the linear equation. After the comparisons, they are estimations of the systematic measurement error components (biases) by each measurement standard.

As additive and multiplicative degrees of equivalence are generalized characteristics of each measurement standard, then correlation appears among the KCRV through them. Mathematically strict processing by LSM demands formulation and solution of the large number of equations. For solving the above mentioned problems the special equations formulated below and in ${ }^{[2,3]}$ are added to them. Formulating and solving the large number of equations is possible only using developed specialized software, which is called «Metrology Network».

Processing measurement results during the key comparisons EURAMET.L-K1.2011 «Measurement of gage blocks by interferometer» formulated in ${ }^{[4]}$ was provided using particular modification according to ${ }^{[1]}$. On the purpose to demonstrate advantages and potential of new mathematical apparatus ${ }^{[3]}$ below are represented results of the repeated processing of the measurement results during comparisons [4] using «Metrology Network» software developed according to ${ }^{[3]}$. Moreover, the comparison of simulated measurement results, which volume is seven times bigger than the measurement results according to ${ }^{[4]}$ was provided to demonstrate comparison opportunies of any big 
volume and complicated measurement combinations. This was provided to ground the opportunities of international comprehensive comparisons arrangement with an expanded group of participants (hereinafter - comprehensive comparisons), which will include measurement standards of almost all national metrological institutions by certain type of measurement if appropriate. As the types of measurements with comparisons deficiency exist, then the idea to provide international comparisons is relevant.

Similar measurement models are well known. For example, a similar model is quite fully described in ${ }^{[5]}$, but it is not modified for processing the results of key comparisons there. It does not include KCRV and does not apply the condition under which the sums of multiplicative and/or additive parameters of the measurement standards are equal to zero.

According to our point of view, absence of the accepted by metrological society simple and understandable mathematical apparatus realized in software is one of the main reasons for international comprehensive comparisons absence.

Moreover, general methodology of measurements adjustment by LSM realization allows solving of methodically the same and mathematically strict plenty of partial cases in the process of regional and reciprocal additional comparisons and any calibrations by the same «Metrology Network» software. Thus, the estimated KCRV such as additive and multiplicative measurement standards degrees of equivalence, as the highest rank of the metrological traceability chain may be used as the reference values for any further comparisons and calibrations. In other words, «Metrology Network» software may realize the metrological traceability chain completely by itself.

\section{General procedure of the key comparisons EURAMET.L-K1.2011 «Measurement of gage blocks by in- terferometer»}

Key comparisons EURAMET.L-K1.2011 «Measurement of gage blocks by interferometer» are described in ${ }^{[4]}$. Twenty-four laboratories participated in these comparisons. They were divided into two groups. The first group participated only in the loop A and the second one - in the loop B. Only three laboratories were the linking and participated in both loops A and B, they are BEV (Austria), METAS (Switzerland) and MIKES (Finland).

Six gage block sets were used in comparisons: two sets consist of eight short steel gage blocks with nominal length from 0,5 to $100 \mathrm{~mm}$; two sets consisted of eight short ceramic gage blocks with nominal length from 0,5 to $100 \mathrm{~mm}$ and two sets consisted of three long steel gage blocks. Three of six sets were used in each of the measurement loops.

«Metrology Network» software repeatedly processed only measurement results of two steel sets and two ceramic sets of short gage blocks.

Consequently, measurement results only of 21 laboratories for steel gage blocks and measurement results only of 20 laboratories for ceramic gage blocks were considered applicable for final processing.

\section{Brief review of the mathematical apparatus for measurement results processing by «Metrology Net- work» software}

«Metrology Network» software is based on the measurement model, which is different from the model defined in the report on key comparison ${ }^{[4]}$. It is defined in ${ }^{[3]}$ and is the following:

$$
x_{i j}=y_{i}+d_{j}+b_{j} \cdot x_{i j}
$$

where

$x_{i j}$ is length value of the gage block measured by the measurement standard of the comparisons participant;

$y_{i}$ is the KCRV value assigned to gage block according to processing results;

$d_{j}, b_{j}$ are additive and multiplicative parameters of the measurement standard, for example additive and multiplicative degrees of equivalence; $i=1 \ldots n$ is gage block number and $j=1 \ldots k$ is measurement standard number correspondingly.

Each measurement standard additive and multiplicative degrees of equivalence are estimations of 
systematic measurement error components by these measurement standards (i.e. they are biases).

For better understanding of the adjustment process, let us transform measurement model equation (1) into correction equation. Omitting some intermediate considerations let us formulate correction equation:

$$
v_{x_{i j}}=\delta y_{i}+\delta d_{j}+\delta b_{j} \cdot x_{i j}+l_{i j}
$$

where

$v_{x_{i j}}$ is correction from adjustments to measured values of gage block length;

$\delta y_{i}, \delta d_{j}, \delta b_{j}$ are correction from adjustments to the initial KCRV and the initial values of additive and multiplicative measurement standard degrees of equivalence;

$l_{i j}=y_{0 i}+d_{0 j}+b_{0 j} \cdot x_{i j}-x_{i j}$ is the constant member of correction equation;

$y_{0 i}, d_{0 j}, b_{0 j}$ are the initial KCRV and the initial values of additive and multiplicative measurement standard degrees of equivalence; ? .

Corrections from adjustments to measured values of gage blocks length (2) actually are each standards degrees of equivalence in each measurement point $v_{x_{i j}}=d_{i j}$. This corresponds to the basic publication ${ }^{[1]}$. Measurement results of the most comparisons are processed using formulae from it.

Necessary quality of «Metrology Network» software is that measurement model (1) is easily transformed to the following partial cases:

$$
\begin{gathered}
x_{i j}=y_{i} ; \quad v_{x_{i j}}=\delta y_{i}+l_{i j} ; \\
x_{i j}=y_{i}+d_{j} ; \quad v_{x_{i j}}=\delta y_{i}+\delta d_{j}+l_{i j} ; \\
x_{i j}=y_{i}+b_{j} \cdot x_{i j} ; \quad v_{x_{i j}}=\delta y_{i}+\delta b_{j} \cdot x_{i j}+l_{i j} .
\end{gathered}
$$

Model (3) actually is the elementary model of the KCRV estimation as weighted average without estimation of the systematic measurement error components. Besides, a combined solution is absent. It is divided into separate averaging. Thus for «Metrology Network» software user it is unnoticeable. This mode may be used to check the effect of the systematic measurement error components exclusion, if more difficult measurement models are used.

Additive degree of equivalence was estimated for each measurement standard according to model (4) separate for steel and ceramic gage blocks. In fact, it is constant, which is added with its sign to each measured length of gage block on the mentioned measurement standard and is an estimation of the systematic measurement error component.

For each measurement standard the additive and multiplicative degrees of equivalence were estimated according to the full model (1) separately for steel and ceramic gage blocks. Thus, both some constant for each measurement standard and some coefficient determining dependence between the measurement error by the measurement standard and measured deviations of the gage blocks length from its nominal values.

Model (5) was not used, as it was proved ineffective in this particular case.

Taking into consideration that the key comparisons are processed and any reference values are absent, then it is necessary to use a free case of comparison with additional summary equations according to ${ }^{[3]}$. Additional summary equations:

$$
\sum_{j=1}^{k} \bar{w}\left(d_{j}\right) \cdot d_{j}=0 ; \quad \sum_{j=1}^{k} \bar{w}\left(b_{j}\right) \cdot b_{j}=0
$$

where

$\bar{w}\left(d_{j}\right)$ and $\bar{w}\left(b_{j}\right)$ are a priori weight of the measurement standard additive and multiplicative degrees of equivalence.

In that particular case of repeated processing of the comparisons results ${ }^{[4]}$ all the degrees of equivalence a priori weights were considered equal. Thus, the simplified equation (6) will be the following:

$$
\sum_{j=1}^{k} d_{j}=0 ; \quad \sum_{j=1}^{k} b_{j}=0 .
$$

«Metrology Network» software adds equation (7) to the system of analogical correction equations to (2). As a result, for solutions formulated below the first condition (7) is executed for both conditions. The first 
necessary condition is that the same average zero is used for all measurements according to the measurement scale. The second necessary condition is that the same average measurement unit is used for all measurements according to the measurement scale. Relative to them the measurement standard additive and multiplicative degrees of equivalence are calculated as estimations of the systematic measurement errors provided by them.

Standard deviation of the gage block length measurement, which weight is equal to the unit is calculated by a formula:

$$
S=\sqrt{\frac{\sum_{i=1}^{n} \sum_{j=1}^{k} w_{i j} \cdot v_{x_{i j}}^{2}}{r}}=\sqrt{\frac{\sum_{i=1}^{n} \sum_{j=1}^{k} w_{i j} \cdot d_{i j}^{2}}{r}},
$$

where

$v_{x_{i j}}=d_{i j}$ is each measurement standards in each point degrees of equivalence;

$r=n \cdot k-n$ is the degrees of freedom maximum quantity for the elementary model (3);

$r=n \cdot k-n-k+1$ is the degrees of freedom maximum quantity for the simplified models (4) and (5);

$r=n \cdot k-n-2 \cdot k+2$ is the degrees of freedom maximum quantity for the full model (1); $w_{i j}=\frac{\sigma_{0}^{2}}{u\left(x_{i j}\right)^{2}}$ is the measurement weight;

$\sigma_{0}^{2}$ is the measured value of gage block dispersion, which weight is considered equal to the unit; $u\left(x_{i j}\right)^{2}$ is the measured length of gage block dispersion $x_{i j}$.

Actually, the degrees of equivalence squares sum is minimized according to the LSM $\sum_{i=1}^{n} \sum_{j=1}^{k} w_{i j} \cdot v_{x_{i j}}^{2}=\sum_{i=1}^{n} \sum_{j=1}^{k} w_{i j} \cdot d_{i j}^{2}=$ min by each measurement standard in each measurement point multiplied on their weights. Average or weighted average calculated by ${ }^{[1]}$ corresponds to the same condition.

Value $S$ is shortly called standard deviation of measurement unit. It characterizes measurement uncertainty of comparison in whole. Its decreasing in the process of comparison characterizes minimization of the systematic measurement error components influ- ence on the final comparison results dependent on the obtaining measurement model.

Type A uncertainty for measurement values assigned to the measurement subjects according to comparisons results of the measurements, subjects (standards) additive and multiplicative degrees of equivalence are calculated by the formulae:

$$
\begin{gathered}
u_{A}\left(y_{i}\right)=S \cdot \sqrt{Q_{y_{i j}}} ; \quad u_{A}\left(d_{j}\right)=S \cdot \sqrt{Q_{d_{j j}}} ; \\
u_{A}\left(b_{j}\right)=S \cdot \sqrt{Q_{b_{j j}}},
\end{gathered}
$$

where

$Q_{y_{i i}}, Q_{d_{j j}}$ and $Q_{b_{j j}}$ are corresponding diagonal members of the matrix inversed to the matrix of normal equations, which include all correlation connections, which appeared.

For estimation of the measurement bias importance by the measurement standards, it is offered to use some coefficient like normalized deviation according to ${ }^{[4]}$ (formula (36)). Difference between offered coefficient (10) from formulated in ${ }^{[4]}$ consists in the fact that as denominator was taken doubled type A standard uncertainty received from adjustment by LSM using formulae (9) instead of expanded uncertainty (doubled standard summary uncertainty):

$$
E_{n(d)}=\frac{d_{j}}{2 \cdot u_{A}\left(d_{j}\right)} ; \quad E_{n(b)}=\frac{b_{j}}{2 \cdot u_{A}\left(b_{j}\right)} .
$$

Normalized deviation (10) is not calculated by modulus as it is done in ${ }^{[4]}$. Length drift (length change) of gage block with time was not calculated by «Metrology Network» software. Unfortunately, this software version does not include such an opportunity, but it is planned in future.

\section{5. «Metrology Network» software short introduction}

«Project» is created in «Metrology Network» software for the solution of the formulated below and analogical tasks connected with processing of measurement results during the comparison process. For comparison of measurement results in the process 
of comparisons, which is considered, «EURAMET.LK1.2011» project was created. It consists of separate files called «Solutions». For example, measurement results of steel and ceramic gage blocks processing is formulated as separate «Solutions». Comparison of measurement results for three laboratories connected by loops of measurements and for all laboratories, which results were accepted for processing, are formulated as separate «Solutions».

In terms of each «Solution», it is easily possible to change software settings, for example to choose a measurement model (1), (3), (4) or (5). It was done during the repeated processing. Clicking on two keys is possible to duplicate any solution, add something new or delete uncertain measurement results. After this, it is possible to save everything as a new «Solution» and provide adjustment.

Each «Solution» of «Metrology Network» software consists of three Tables. Input data for comparisons is entered to the Tables and adjustment results are received from them.

First Table includes measurement results. It consists of six columns:

- $\quad$ name of the measurement subject;

- $\quad$ name of the measurement object;

- measured quantity value;

- $\quad$ standard measurement uncertainty of this value;

- correction to measured quantity value, which is the degree of equivalence in the point of measurement;

- $\quad$ adjusted value consists of measured one plus correction.

Entering of the input data to the Table may be provided from «Word» or «Excel» tables using standard commands «Ctrl C», «Ctrl V», the same as for «Metrology Network» software information transfer to «Word» or «Excel» files.

Measurement subjects mean measurement standards, realizing or reproducing measurement unit and measurement objects mean artefacts, to which reference values are assigned as calculations results. All measurements provided by measurement subjects on measurement objects for this particular «Solution» are entered into this Table.

The second Table includes measurement objects. It consists of six columns:

- $\quad$ name of the measurement object;

- initial quantity value assigned to the measurement object before the adjustments;

- $\quad$ standard uncertainty of the quantity value before the adjustments (theoretically may be equal from zero to infinity);

correction to quantity value from the adjustments;

- adjusted quantity value, assigned to the measurement subject after the adjustments;

- $\quad$ standard uncertainty of the adjusted quantity value after the adjustments.

The third Table includes measurement subjects. It also consists of six columns. For each measurement subject was defined its name and two rows of six columns each one. Separate column for additive and separate column for multiplicative parameter of the measurement subject.

For each row:

- $\quad$ check box to mark additive and/or multiplicative parameter or none of them can be marked (model (1) or (4) or (5) or (3) is used);

- initial value assigned to this parameter before the adjustment;

- standard uncertainty of parameter before the adjustments (theoretically may be equal from zero to infinity);

- correction to input parameter value from adjustments;

- adjusted parameter value, assigned to the measurement subject after the adjustments;

- standard uncertainty of the adjusted parameter value after the adjustments.

Names of objects and subjects entered to the first Table are automatically duplicated in the second and third Tables. There is no other way to enter new measurement objects and subjects. 
On default, uncertainty of all quantity values for measurement objects or parameters for measurement subjects before the adjustments is relatively considered equal to infinity. This status is denoted by the word «Free», thus it should be estimated by results of adjustments. If all values and parameters have «Free» status, then the solution has «Free» status also ${ }^{[3]}$.

While having the necessity of a solution strict binding to one or few quantity values or parameters, then the status of those values is changed to «Fixed». This means that assigned to fixed values uncertainty is equal to zero and in the process of normal equations solving their values will not get the corrections and all other adjusted values will be bounded to fixed ones considering all correlation links. If at least one value or parameter is fixed, then the solution will have «Fixed» status also ${ }^{[3]}$.

Additive and multiplicative parameters of measurement subjects may be included or excluded, fixed or free in any combination. In any combination with them may be fixed or free quantity values assigned to measurement objects as well.

Intermediate adjustment between «Free» and «Fixed» may be provided. One, several or all quantity values assigned to measurement objects or parameters of subjects have uncertainty comparable to measurement uncertainty. Adjustment in that case refers to those values but changes them using new measurements. Such values have «Dependent» status. If at least one has «Dependent» status among those, which have «Free» status, then the solution will have «Dependent» status also. If at least one has «Fixed» status among those, which have «Dependent» status, then the solution will have «Fixed» status also.

Quantity values assigned to the objects, additive and multiplicative parameters of measurement subjects may have any uncertainty in any combination with included or excluded, fixed or free quantity values and parameters.

Pseudo-inverted matrix of normal equations is always used in «Metrology Network» software for normal equations solving according to ${ }^{[6]}$. This slightly slows down the process of adjustment by «Metrology Network» software, but frees from the necessity to check if the matrix of the normal equations degenerates in the process of inversion.

\section{Analyses of measurement standard additive de- grees of equivalence as estimations of the systematic measurement error components by them}

Three laboratories BEV, METAS and MIKES according to ${ }^{[4]}$ provided measurements in two loops A and B for both steel and ceramic gage blocks. Thus, linking of all measurements for two loops is provided through their measurements. The decision to process these measurements separately was made considering their importance.

As it was mentioned above the adequacy criteria of used measurement model to any measurement results is standard measurement deviation of weight unit $S$. If a more complicated model provides a quantity value of standard deviation considerable decrease, it means that the measurement model is adequate and some estimated parameters of the model are estimations of the relevant systematic measurement error components.

For elementary model (3) the following estimations of standard deviation of weight unit are 12,8 nm for steel gage blocks and $8,3 \mathrm{~nm}$ for ceramic gage blocks. After the adjustment using simplified model (4) these quantity values decreased to $9,5 \mathrm{~nm}$ and $5,4 \mathrm{~nm}$ that is $26 \%$ and $34 \%$ correspondingly. This difference is rather considerable.

Processing results by simplified model (4) and condition (7) are represented in Table 1. Standard type A uncertainties in Table 1 and the following ones are calculated by the formulae (9) and normalized deviations $E_{n(d)}$ by the formula (10).

The following conclusions may be done according to Table 1:

1. Measurement standard additive degrees of equivalence, which are estimations of the systematic measurement error components of gage blocks length measurement by measurement standards (biases) are very considerable for two standards of three.

2. For these three laboratories this systematic measurement error component does not depend on 
Table 1. Measurement standard additive degrees of equivalence, which are linking between two loops

\begin{tabular}{|l|c|c|c|c|c|c|c|}
\hline \multirow{2}{*}{ Laboratory } & \multicolumn{3}{|c|}{ Steel gage blocks } & \multicolumn{3}{c|}{ Ceramic gage blocks } & \multirow{2}{*}{\begin{tabular}{c} 
Difference \\
\cline { 2 - 7 }
\end{tabular}} \\
\cline { 2 - 7 } & $d_{j}, \mathrm{~nm}$ & $u_{A}\left(d_{j}\right), \mathrm{nm}$ & $E_{n(d)}$ & $d_{j}, \mathrm{~nm}$ & $u_{A}\left(d_{j}\right), \mathrm{nm}$ & $E_{n(d)}$ & $\delta$ \\
\hline BEV & 8.7 & 3.5 & 1.24 & 6.5 & 2.4 & 1.35 & 2.2 \\
\hline METAS & -11.7 & 3.1 & 1.92 & -9.3 & 2.3 & 2.02 & -2.4 \\
\hline MIKES & 3.0 & 3.2 & 0.47 & 2.8 & 2.2 & 0.64 & 0.2 \\
\hline
\end{tabular}

the production material of gage block. Quantity values are close for both steel and ceramic gage blocks.

3. Measurement results by three laboratories are completely reliable for linking measurement results in different loops, nevertheless, taking into account systematic measurement error component according to model (4) is necessary.

Additive degrees of equivalence calculated by model (4) for all comparisons participants standards included for processing are represented in Table 2 . Calculation results for steel and ceramic gage blocks are represented separately as in Table 1. Data from 21 laboratories, which measured steel gage blocks length and 20 laboratories, which measured ceramic gage blocks, was completely processed out of 24 laboratories announced for comparisons.

For elementary model (3) the following estimations of standard deviation of weight unit are $12,8 \mathrm{~nm}$ for steel gage blocks and 13,8 nm for ceramic gage blocks. After the adjustment using simplified model (4) these quantity values decreased to $10,5 \mathrm{~nm}$ and $10,1 \mathrm{~nm}$ that is $18 \%$ and $27 \%$ correspondingly. This difference is rather considerable.

A priori standard deviation of gage blocks length measurement, which was used as numerator in formula of measurement weights calculation (8) and for which the weight was considered equal to the unit, that is $\sigma_{0}=10 \mathrm{~nm}$. This is very close to experimental standard deviations after the measurement results of steel and ceramic gage blocks length adjustments, particularly $10,5 \mathrm{~nm}$ and $10,1 \mathrm{~nm}$. This fact means that measurement participants on average estimated their own measurement uncertainties adequately. The best convergence was received only after additive degrees of equivalence were excluded from estimation of standard uncertainty of weight unit according to (8) in the process of adjustment by model (4).

Prior analyses of corrections form adjustments (2) to measurement results of steel gage blocks length by three linking laboratories demonstrated that standard deviation after adjustment by model (4) for BEV is $7,5 \mathrm{~nm}$, for METAS is $8,1 \mathrm{~nm}$ and for MIKES is $13,4 \mathrm{~nm}$. Announced by laboratories, standard measurement uncertainties in the length of gage blocks range from 0,5 $\mathrm{nm}$ to $100 \mathrm{~nm}$ are for BEV $15 \mathrm{~nm}$, for METAS from 9,5 to $13 \mathrm{~nm}$ and for MIKES from 10 to $18 \mathrm{~nm}$. This proves the possibility to consider the question of analyzing adequacy of announced estimations of measurement uncertainties by the laboratories compared to statistical estimations received in the process of adjustment. «Metrology Network» may easily realize this function.

The following conclusions may be done according to Table 2:

1. For the first group of participants, measurement standards additive degrees of equivalence received for steel and ceramic gage blocks are close enough (upper part of Table 2). This fact is positive. For the second group (lower part of Table 2) difference is considerable and should be the subject of analyses by the laboratories.

2. Fifteen of forty one of measurement standards degrees of equivalence exceed its doubled standard uncertainty (coefficient $-1 \geq E_{n} \geq 1$ ). For other four coefficient is $-0,9 \geq E_{n} \geq 0,9$. It means that for more than the third part of measurement standards measurement bias of gage blocks length is considerable. 
Table 2. Additive degrees of equivalence for all comparisons participants standards included for processing

\begin{tabular}{|c|c|c|c|c|c|c|c|c|}
\hline \multirow{2}{*}{ Loop } & \multirow{2}{*}{ Laboratory } & \multicolumn{3}{|c|}{ Steel gage blocks } & \multicolumn{3}{|c|}{ Ceramic gage blocks } & \multirow{2}{*}{$\begin{array}{l}\text { Difference } \\
\delta d_{j}, \mathrm{~nm}\end{array}$} \\
\hline & & $d_{j}, \mathrm{~nm}$ & $u_{A}\left(d_{j}\right), \mathrm{nm}$ & $E_{n(d)}$ & $d_{j}, \mathrm{~nm}$ & $u_{A}\left(d_{j}\right), \mathrm{nm}$ & $E_{n(d)}$ & \\
\hline \multicolumn{9}{|c|}{ First group of participants } \\
\hline $\mathrm{AB}$ & BEV & 8.9 & 4.0 & 1.11 & 6.8 & 3.8 & 0.90 & 2.1 \\
\hline B & CMI & 1.1 & 5.0 & & 5.3 & 5.2 & & -4.2 \\
\hline $\mathrm{AB}$ & MIKES & 2.9 & 3.1 & & 3.2 & 2.9 & & -0.3 \\
\hline B & GUM & 2.6 & 5.0 & & 0.3 & 4.4 & & 2.3 \\
\hline B & SMD & 3.7 & 4.0 & & -1.0 & 3.4 & & 4.7 \\
\hline B & LNE & -1.7 & 3.8 & & -4.4 & 3.7 & & 2.7 \\
\hline B & VSL & 3.1 & 4.4 & & -2.8 & 3.9 & & 5.9 \\
\hline B & NPL & -3.7 & 4.4 & & -7.4 & 4.2 & -0.90 & 3.7 \\
\hline A & EIM & -2.5 & 5.2 & & -8.3 & 5.0 & & 5.8 \\
\hline $\mathrm{AB}$ & METAS & -11.8 & 2.9 & -2.03 & -9.0 & 3.2 & -1.40 & -2.8 \\
\hline B & CEM & -17.2 & 4.3 & -2.00 & -22.1 & 4.0 & -2.76 & 4.9 \\
\hline \multicolumn{9}{|c|}{ Second group of participants } \\
\hline A & DMDM & -3.9 & 4.4 & & 16.2 & 4.3 & 1.88 & 20.1 \\
\hline A & SMU & 25.0 & 5.1 & 2.45 & - & - & - & - \\
\hline A & UME & -0.8 & 5.4 & & 22.7 & 5.1 & 2.23 & -23.5 \\
\hline A & NIS & -20.0 & 10.9 & -0.92 & -7.0 & 8.0 & & -13.0 \\
\hline A & DFM & 11.8 & 5.1 & 1.16 & -6.5 & 4.9 & & 18.3 \\
\hline A & FSB & -13.2 & 6.2 & -1.06 & -27.7 & 6.1 & -2.27 & 14.5 \\
\hline A & $\mathrm{JV}$ & -11.3 & 4.5 & -1.26 & -3.2 & 4.5 & & -8.1 \\
\hline B & INM & 12.8 & 6.8 & 0.94 & 22.1 & 6.3 & 1.75 & -9.3 \\
\hline B & SP & -1.7 & 5.8 & & -18.9 & 5.7 & -1.66 & 17.2 \\
\hline B & IPQ & 15.8 & 10.5 & & 41.7 & 9.7 & 2.15 & -25.9 \\
\hline
\end{tabular}

3. Only for six measurement standards CMI, MIKES, GUM, SMD, LNE, VSL measurement biases may be accepted as not considerable for both steel and ceramic gage blocks simultaneously.

4. Differences between additive degrees of equivalence (biases) for steel and ceramic gage blocks represented in the last column of Table 2 mean their constant character for measurement standards of the first group. Thus, only four of 11 exceed standard measurement deviations of the biases. For the second group differences are very considerable, nevertheless, they exceed their standard deviations several times. 
5. Constant character of measurement biases by each measurement standard of the first group gives the opportunity to use them as corrections in the process of calibration and as reference values for further comparisons.

7. Combined analysis of measurement standards additive and multiplicative degrees of equivalence

In the process of adjustment according to the measurement model (1) approximation was provided by linear function of the measurement errors by each measurement standard together with the KCRV calculation. Measurement standards additive and multiplicative degrees of equivalence actually are these coefficients of approximating linear functions. In the previous section, additive degree of equivalence was proved to have constant character for many laboratories. The aim of this section is to check if the multiplicative degree of equivalence has the same constant character according to the comparisons results ${ }^{[4]}$. Moreover, it is necessary to check how it interacts with the additive one.

As it was mentioned above, model (4) provided estimation of standard measurement deviation of weight unit for steel gage blocks $10,5 \mathrm{~nm}$. After the adjustment by full model (1) this value decreased to $8,4 \mathrm{~nm}$, that is $20 \%$. This is rather considerable.

Model (1) compared to model (4) for ceramic gage blocks provided a slight increase of standard measurement deviation of weight unit from $10,1 \mathrm{~nm}$ to $10,4 \mathrm{~nm}$. This proves full model (1) to be not better than the simplified (4) in this particular case.

In Tables 3 and 4, separately additive and multiplicative degrees of equivalence are represented for the comparisons participants measurement standards received by the full model (1).

«Metrology Network» software calculates multiplicative degrees of equivalence in this particular case of adjustment, expressed as a coefficient of linear equation in nanometers by micrometer $(\mathrm{nm} / \mu \mathrm{m})$. For example, if

Table 3. Additive degrees of equivalence for all comparisons participants standards included for processing by model (1)

\begin{tabular}{|c|c|c|c|c|c|c|c|c|}
\hline \multirow{2}{*}{ Loop } & \multirow{2}{*}{ Laboratory } & \multicolumn{3}{|c|}{ Steel gage blocks } & \multicolumn{3}{|c|}{ Ceramic gage blocks } & \multirow{2}{*}{$\begin{array}{l}\text { Difference } \\
\delta d_{j}, \mathrm{~nm}\end{array}$} \\
\hline & & $d_{j}, \mathrm{~nm}$ & $u_{A}\left(d_{j}\right), \mathrm{nm}$ & $E_{n(d)}$ & $d_{j}, \mathrm{~nm}$ & $u_{A}\left(d_{j}\right), \mathrm{nm}$ & $E_{n(d)}$ & \\
\hline \multicolumn{9}{|c|}{ First group of participants } \\
\hline A B & BEV & 10.5 & 3.5 & 1.50 & 7.0 & 5.6 & & 3.5 \\
\hline B & CMI & 2.8 & 4.1 & & 4.7 & 8.9 & & -1.9 \\
\hline A B & MIKES & 2.1 & 2.6 & & 2.6 & 4.3 & & -0.5 \\
\hline B & GUM & 5.0 & 4.1 & & -0.6 & 7.2 & & 5.6 \\
\hline B & SMD & 4.1 & 3.4 & & -0.8 & 4.4 & & 4.9 \\
\hline B & LNE & -0.9 & 3.2 & & -2.9 & 6.1 & & 2.0 \\
\hline B & VSL & 2.2 & 3.7 & & -5.8 & 6.3 & & 8.0 \\
\hline B & NPL & -2.4 & 3.7 & & 4.3 & 7.2 & & -6.7 \\
\hline A & EIM & -8.5 & 4.7 & -0.90 & -10.4 & 5.9 & & 1.9 \\
\hline A B & METAS & -10.5 & 2.5 & -2.10 & -10.5 & 4.2 & -1.25 & 0.0 \\
\hline B & CEM & -12.5 & 3.5 & -1.78 & -18.3 & 6.2 & -1.48 & 5.8 \\
\hline
\end{tabular}


Measurements infrastructure Vol. 2 (2021) MI_009_120521

Table 3. Additive degrees of equivalence for all comparisons participants standards included for processing by model (1). (Continuation)

\begin{tabular}{|c|c|c|c|c|c|c|c|c|}
\hline \multirow{2}{*}{ Loop } & \multirow{2}{*}{ Laboratory } & \multicolumn{3}{|c|}{ Steel gage blocks } & \multicolumn{3}{|c|}{ Ceramic gage blocks } & \multirow{2}{*}{$\begin{array}{c}\text { Difference } \\
\delta d_{j}, \mathrm{~nm}\end{array}$} \\
\hline & & $d_{j}, \mathrm{~nm}$ & $u_{A}\left(d_{j}\right), \mathrm{nm}$ & $E_{n(d)}$ & $d_{j}, \mathrm{~nm}$ & $u_{A}\left(d_{j}\right), \mathrm{nm}$ & $E_{n(d)}$ & \\
\hline \multicolumn{9}{|c|}{ Second group of participants } \\
\hline A & DMDM & -5.0 & 4.0 & & 19.2 & 6.0 & 1.88 & 24.2 \\
\hline A & SMU & 17.2 & 4.4 & 1.95 & - & - & & - \\
\hline A & UME & 1.5 & 4.9 & & 24.4 & 7.3 & 1.67 & -22.9 \\
\hline A & NIS & -28.4 & 9.7 & -1.46 & -9.2 & 9.2 & & -19.2 \\
\hline A & DFM & 10.4 & 4.3 & 1.21 & -8.1 & 5.8 & & 18.3 \\
\hline A & FSB & -6.8 & 5.7 & & -29.2 & 6.6 & -2.21 & 22.4 \\
\hline A & $\mathrm{JV}$ & -10.7 & 4.1 & -1.30 & -5.9 & 5.4 & & -4.8 \\
\hline B & INM & 16.2 & 6.8 & 1.19 & 26.7 & 17.6 & & -9.3 \\
\hline B & SP & -3.3 & 4.8 & & -14.5 & 9.0 & & 10.5 \\
\hline B & IPQ & 17.1 & 8.5 & 1.01 & 27.5 & 18.4 & & -10.4 \\
\hline
\end{tabular}

Table 4. Multiplicative degrees of equivalence for all comparisons participants standards included for processing by model (1)

\begin{tabular}{|c|c|c|c|c|c|c|c|}
\hline \multirow{2}{*}{ Loop } & \multirow{2}{*}{ Laboratory } & \multicolumn{3}{|c|}{ Steel gage blocks } & \multicolumn{3}{|c|}{ Ceramic gage blocks } \\
\hline & & $b_{j}, \mathrm{~nm} / \mu \mathrm{m}$ & $\begin{array}{l}u_{A}\left(b_{j}\right), \\
\mathrm{nm} / \mu \mathrm{m}\end{array}$ & $E_{n(b)}$ & $b_{j}, \mathrm{~nm} / \mu \mathrm{m}$ & $\begin{array}{l}u_{A}\left(b_{j}\right), \\
\mathrm{nm} / \mu \mathrm{m}\end{array}$ & $E_{n(b)}$ \\
\hline \multicolumn{8}{|c|}{ First group of participants } \\
\hline A B & $\mathrm{BEV}$ & 44.1 & 19 & 1.16 & -4.0 & 45 & \\
\hline B & CMI & 74.4 & 24 & 1.55 & 10.8 & 82 & \\
\hline A B & MIKES & 1.9 & 20 & & 6.6 & 40 & \\
\hline B & GUM & 74.7 & 24 & 1.56 & 9.3 & 58 & \\
\hline B & SMD & 63.5 & 34 & 0.93 & -15.1 & 43 & \\
\hline B & LNE & 31.6 & 22 & & -15.3 & 58 & \\
\hline B & VSL & 3.8 & 22 & & 34.4 & 53 & \\
\hline B & NPL & 45.9 & 21 & 1.09 & -133 & 67 & -0.99 \\
\hline A & EIM & -71.4 & 38 & -0.94 & 42.4 & 60 & \\
\hline A B & METAS & 35.7 & 18 & 0.99 & 22.1 & 37 & \\
\hline B & CEM & 122.3 & 21 & 5.82 & -54.4 & 68 & \\
\hline
\end{tabular}


Table 4. Multiplicative degrees of equivalence for all comparisons participants standards included for processing by model (1). (Continuation)

\begin{tabular}{|c|c|c|c|c|c|c|c|}
\hline \multirow{2}{*}{ Loop } & \multirow{2}{*}{ Laboratory } & \multicolumn{3}{|c|}{ Steel gage blocks } & \multicolumn{3}{|c|}{ Ceramic gage blocks } \\
\hline & & $b_{j}, \mathrm{~nm} / \mu \mathrm{m}$ & $\begin{array}{l}u_{A}\left(b_{j}\right), \\
\mathrm{nm} / \mu \mathrm{m}\end{array}$ & $E_{n(b)}$ & $b_{j}, \mathrm{~nm} / \mu \mathrm{m}$ & $\begin{array}{l}u_{A}\left(b_{j}\right), \\
\mathrm{nm} / \mu \mathrm{m}\end{array}$ & $E_{n(b)}$ \\
\hline \multicolumn{8}{|c|}{ Second group of participants } \\
\hline A & DMDM & 9.3 & 26 & & -52.8 & 56 & \\
\hline A & SMU & -159 & 40 & -1.99 & - & - & \\
\hline A & UME & 52 & 31 & & -29.5 & 65 & \\
\hline A & NIS & -395 & 221 & 0.90 & 41 & 88 & \\
\hline A & DFM & 2.4 & 37 & & 28.0 & 59 & \\
\hline A & FSB & 107 & 37 & 1.45 & 53.3 & 71 & \\
\hline A & JV & 32.6 & 33 & & 50.9 & 53 & \\
\hline B & INM & -26.2 & 96 & & -44.4 & 167 & \\
\hline B & SP & -24.0 & 28 & & -55.0 & 87 & \\
\hline B & IPQ & -25.3 & 75 & & 105.3 & 116 & \\
\hline
\end{tabular}

this coefficient is equal to $44 \mathrm{~nm} / \mu \mathrm{m}$, then measurement bias by measurement standard is 44 nanometers by one micrometer (1000 nanometers) of the gage block length deviation from nominal. The same measurement units express uncertainty of these coefficients.

According to Tables 3 and 4:

1. The most standards additive degrees of equivalence calculated by simplified model (4) and full model (1) differ noticeably by quantity but changes are not considerable. Thus, standards additive degrees of equivalence are left as constant characteristics for the first group. For the second group they differ considerably.

2. Measurement standards multiplicative degrees of equivalence represented in Table 4 do not have constant character. For most laboratories, they are considerably different for steel and ceramic gage blocks.

3. Uncertainty of multiplicative degrees of equivalence are much better for steel gage blocks than for ceramic one. This is caused by the fact that values of gage blocks deviations from their nominal values for steel gage blocks are evenly distributed in the measurement range and have negative and positive values (Table 5). For ceramic gage blocks this range is shorter and the deviations are clustered (not evenly distributed), mostly having positive value (Table 6).

4. Some laboratories have large additive and/or multiplicative degrees of equivalence and are rather different for steel and ceramic gage blocks in Table 3 and 4. Based on this all measurements provided by these laboratories may be excluded from calculations of the KCRV in the first round and in the second one may be calculated referring to new reference values.

8. Comprehensive analysis of the KCRV from report on key comparisons and according to adjustment by the «Metrology Network» software

In columns 3 and 4 of Tables 5 and $6, \mathrm{KCRV}$ and their standard uncertainties are entered from a report 
on key comparisons ${ }^{[4]}$, correspondingly for steel and ceramic gage blocks.

In columns 5 and 6 of Tables 5 and $6, \mathrm{KCRV}$ and their standard uncertainties are entered based on adjustments results by «Metrology Network» software of three linking laboratories BEV, METAS and MIKES, correspondingly for steel and ceramic gage blocks according to model (4).
In columns 8 and 9 of Tables 5 and 6, KCRV and their standard uncertainties are entered based on adjustments results by «Metrology Network» software of all participating laboratories, which results were allowed to adjustment, correspondingly for steel and ceramic gage blocks according to model (4).

In Table 5 measured length for two steel $100 \mathrm{~mm}$ gage blocks from ${ }^{[4]}$ was calculated taking into account

Table 5. Comparison of the KCRV from report ${ }^{[4]}$ and received on adjustments results by «Metrology Network» software for steel gage blocks according to model (4)

\begin{tabular}{|c|c|c|c|c|c|c|c|c|c|}
\hline \multicolumn{4}{|c|}{ Results of EURAMET.L-K1.2011 from ${ }^{[4]}$} & \multicolumn{6}{|c|}{$\begin{array}{c}\text { Results of adjustment of EURAMET.L-K1.2011 by LSM freedom } \\
\text { case with summary equation }\end{array}$} \\
\hline \multirow[t]{2}{*}{ Loop } & $\begin{array}{l}\text { Nominal } \\
\text { length, } \\
\mathrm{mm}\end{array}$ & $\begin{array}{l}\text { Reference } \\
\text { value, } \mathrm{nm}\end{array}$ & $\begin{array}{l}\text { Standard } \\
\text { uncertainty, } \\
\text { nm }\end{array}$ & $\begin{array}{l}\text { Reference } \\
\text { value, nm }\end{array}$ & $\begin{array}{l}\text { Standard } \\
\text { uncertainty, } \\
\text { nm }\end{array}$ & $\begin{array}{l}\text { Diffe- } \\
\text { rences, } \\
\text { nm }\end{array}$ & $\begin{array}{l}\text { Reference } \\
\text { value, nm }\end{array}$ & $\begin{array}{l}\text { Standard } \\
\text { uncertainty, } \\
\text { nm }\end{array}$ & $\begin{array}{l}\text { Diffe- } \\
\text { rences, } \\
\text { nm }\end{array}$ \\
\hline & $Y_{i}$ & $y_{i}^{E U R}$ & $u\left(y_{i}\right)$ & $y_{i}$ & $u\left(y_{i}\right)$ & $\begin{array}{l}\Delta_{i}= \\
=y_{i}^{E U R}-y_{i}\end{array}$ & $y_{i}$ & $u\left(y_{i}\right)$ & $\begin{array}{l}\Delta_{i}= \\
=y_{i}^{E U R}-y_{i}\end{array}$ \\
\hline 1 & 2 & 3 & 4 & 5 & 6 & 7 & 8 & 9 & 10 \\
\hline \multirow[t]{8}{*}{ A } & 0.5 & -3.8 & 3.5 & 3.7 & 6.4 & -7.5 & -2.7 & 4.1 & -1.1 \\
\hline & 1.15 & -44.5 & 3.2 & -48.8 & 6.4 & 4.5 & -47.4 & 3.7 & 2.9 \\
\hline & 3 & 56.1 & 3.1 & 57.0 & 6.4 & -0.9 & 56.2 & 3.8 & -0.1 \\
\hline & 5 & 21.7 & 3.1 & 22.1 & 6.4 & -0.4 & 21.0 & 3.8 & 0.7 \\
\hline & 7 & -103.2 & 3.2 & -105.6 & 6.4 & 2.4 & -102.3 & 4.2 & -0.9 \\
\hline & 23.5 & -99.6 & 3.6 & -101.9 & 6.7 & 2.3 & -102.0 & 3.9 & 2.4 \\
\hline & 80 & -244.1 & 5.3 & -246.3 & 8.5 & 2.2 & -244.2 & 5.8 & 0.1 \\
\hline & 100 & -540.6 & 6.0 & -539.9 & 8.5 & -0.7 & -534.7 & 6.4 & -5.9 \\
\hline \multirow[t]{8}{*}{ B } & 0.5 & 34.8 & 3.2 & 38.0 & 6.4 & -3.2 & 37.2 & 3.8 & -2.4 \\
\hline & 1.15 & 25.2 & 3.1 & 24.9 & 6.4 & 0.3 & 26.8 & 3.8 & -1.6 \\
\hline & 3 & 25.5 & 3.3 & 30.4 & 6.4 & -4.9 & 28.2 & 3.9 & -2.7 \\
\hline & 5 & -70.1 & 3.1 & -71.1 & 6.4 & 1.0 & -68.0 & 3.8 & -2.1 \\
\hline & 7 & 34.4 & 3.2 & 32.0 & 6.4 & 2.4 & 36.0 & 3.8 & -1.6 \\
\hline & 23.5 & 134.8 & 3.4 & 135.8 & 6.7 & -1.0 & 136.7 & 4.0 & -1.9 \\
\hline & 80 & -332.4 & 4.6 & -330.1 & 8.4 & -2.3 & -331.1 & 5.2 & -1.1 \\
\hline & 100 & -714.9 & 5.7 & -714.7 & 9.0 & -0.2 & -727.7 & 6.0 & 12.8 \\
\hline
\end{tabular}


linear drift for time range equal to average among the measurement time in BEV, METAS and MIKES (q.v. column 3 in Table 5).

In columns 7 and 10 of Tables 5 and 6 differences of KCRV from ${ }^{[4]}$ and calculated results by «Metrology Network» software are entered. Based on the analysis of differences, the conclusion is that no strict coincidence of KCRV is found, though no considerable dif- ferences are found as well. Among 64 analyzed results, only 2 difference values as absolute value exceeded doubled standard uncertainty of reference value from adjustment. Some more difference values mostly for ceramic gage blocks exceed standard uncertainty of the reference value from adjustment.

This proves that KCRV are made more accurate due to weakening of the estimated systematic

Table 6. Comparison of KCRV from report ${ }^{[4]}$ and received on adjustments results by «Metrology Network» software for ceramic gage blocks according to model (4)

\begin{tabular}{|c|c|c|c|c|c|c|c|c|c|}
\hline \multicolumn{4}{|c|}{ Results of EURAMET.L-K1.2011 from ${ }^{[4]}$} & \multicolumn{6}{|c|}{$\begin{array}{l}\text { Results of adjustment of EURAMET.L-K1.2011 by LSM freedom } \\
\text { case with summary equation }\end{array}$} \\
\hline \multirow[t]{2}{*}{ Loop } & $\begin{array}{l}\text { Nominal } \\
\text { length, } \\
\text { mm }\end{array}$ & $\begin{array}{l}\text { Reference } \\
\text { value, } \mathrm{nm}\end{array}$ & $\begin{array}{l}\text { Standard } \\
\text { uncertainty, } \\
\text { nm }\end{array}$ & $\begin{array}{l}\text { Reference } \\
\text { value, nm }\end{array}$ & $\begin{array}{l}\text { Standard } \\
\text { uncertainty, } \\
\text { nm }\end{array}$ & $\begin{array}{l}\text { Diffe- } \\
\text { rences, } \\
\mathrm{nm}\end{array}$ & $\begin{array}{l}\text { Reference } \\
\text { value, nm }\end{array}$ & $\begin{array}{l}\text { Standard } \\
\text { uncertainty, } \\
\text { nm }\end{array}$ & $\begin{array}{l}\text { Diffe- } \\
\text { rences, } \\
\text { nm }\end{array}$ \\
\hline & $Y_{i}$ & $y_{i}^{E U R}$ & $u\left(y_{i}\right)$ & $y_{i}$ & $u\left(y_{i}\right)$ & $\begin{array}{l}\Delta_{i}= \\
=y_{i}^{E U R}-y_{i}\end{array}$ & $y_{i}$ & $u\left(y_{i}\right)$ & $\begin{array}{l}\Delta_{i}= \\
=y_{i}^{E U R}-y_{i}\end{array}$ \\
\hline 1 & 2 & 3 & 4 & 5 & 6 & 7 & 8 & 9 & 10 \\
\hline \multirow[t]{8}{*}{ A } & 0.5 & 48.6 & 3.3 & 53.2 & 4.1 & -4.6 & 48.9 & 3.8 & -0.3 \\
\hline & 1.15 & 135.2 & 3.3 & 139.2 & 4.1 & -4.0 & 135.3 & 3.8 & -0.1 \\
\hline & 3 & 83.5 & 3.3 & 84.7 & 4.1 & -1.2 & 83.7 & 3.8 & -0.2 \\
\hline & 5 & 79.0 & 3.3 & 83.1 & 4.1 & -4.1 & 79.3 & 3.8 & -0.3 \\
\hline & 7 & -130.5 & 3.5 & -126.2 & 3.9 & -4.3 & -126.4 & 3.7 & -4.1 \\
\hline & 23.5 & 41.8 & 3.7 & 34.2 & 4.3 & 7.6 & 39.3 & 4.1 & 2.5 \\
\hline & 80 & 158.9 & 5.2 & 147.7 & 5.0 & 11.2 & 153.0 & 5.5 & 5.9 \\
\hline & 100 & -10.8 & 5.6 & -17.7 & 5.3 & 6.9 & -12.5 & 5.9 & 1.7 \\
\hline \multirow[t]{8}{*}{ B } & 0.5 & 64.7 & 3.1 & 68.7 & 4.1 & -4.0 & 68.3 & 3.6 & -3.6 \\
\hline & 1.15 & 131.3 & 3.2 & 131.6 & 4.1 & -0.3 & 135.2 & 3.7 & -3.9 \\
\hline & 3 & 49.1 & 3.4 & 51.9 & 4.1 & -2.8 & 48.5 & 3.8 & 0.6 \\
\hline & 5 & 52.7 & 3.2 & 54.1 & 4.1 & -1.4 & 56.5 & 3.6 & -3.8 \\
\hline & 7 & 76.4 & 3.3 & 78.3 & 4.1 & 2.4 & 81.1 & 3.8 & -4.7 \\
\hline & 23.5 & 43.1 & 3.3 & 45.7 & 4.3 & -2.6 & 46.0 & 3.8 & -2.9 \\
\hline & 80 & 133.7 & 4.7 & 133.6 & 5.0 & 0.1 & 129.2 & 4.9 & 4.5 \\
\hline & 100 & 330.1 & 5.2 & 334.4 & 5.3 & -4.3 & 325.5 & 5.3 & 4.6 \\
\hline
\end{tabular}


measurement error components influence but in this particular case, differences are acceptable. According to this «Metrology Network» software may be considered applicable for processing of comparisons results.

\section{Short description of future international compre- hensive comparisons}

Processing of 181 length measurement results of steel gage blocks and 178 length measurement results of ceramic gage blocks are represented above. Even processing of such a relatively small quantity of measurement results using general methodology of adjustment by the LSM makes it impossible to use such an accessible instrument as «Excel». Thus, it is connected with formulating and solving several dozens of equations.

Let us imagine future comprehensive comparisons of short gage blocks provided under the aegis of BIPM simultaneously by all regional metrological organizations. Those comparisons were simulated based on the represented above real comparisons. The aim of this simulation is to check «Metrology Network» software capability of processing much larger quantity of the measurement results, linked by cross-measurements.

Comparisons with 120 participating laboratories were simulated. Tasks of finding the possibility to establish a tight measurement schedule for analogical real comparisons for quick passing through them were established. For this purpose, comparisons of 12 steel gage block sets and 12 ceramic gage block sets of 8 gage blocks each were simulated. Thus, 24 measurement loops of 10 laboratories each one were simulated. In each group of 10 laboratories three laboratories were simulated, participating in two related loops for both two steel and two ceramic gage block sets. Thus, the laboratories were combined in groups of 20 each one. Moreover, in each group of 20 laboratories three other laboratories were simulated, which provided measurements of their group sets and of two more steel gage block sets from a related group of 20 laboratories. The total number of processed simulated measurements is 2442. They are similar in structure and statistical characteristics of systematic and random measurement error components to real measurements in key com- parisons process of EURAMET.L-K1.2011 «Measurement of gage blocks by interferometer» represented in ${ }^{[4]}$.

«Metrology Network» software perfectly managed this task. All four measurement models were used in turns, exactly elementary (3), two simplified (4) and (5), when separately additive and multiplicative degrees of equivalence are calculated and full model (1), when they are calculated together. In all models, 192 normal equations were formulated for the KCRV. Up to 120 more normal equations for additive and up to 120 ones for multiplicative degrees of equivalence were formulated for the full model. In total, 432 normal equations for the full model (1) under conditions (7) were formulated and solved. Efficiency criteria for each model use was decrease of standard deviation of the weight unit (8) as previously. Thus, for the elementary model (3) when the solution was actually divided by calculation of separate averaged values, standard deviation of the weight unit was equal to $13,75 \mathrm{~nm}$. As a result of the simplified model (5) use, when only the multiplicative component was calculated, decrease was not considerable up to $12,95 \mathrm{~nm}$. Nevertheless, after use of the simplified model only with additive component, decrease was more considerable up to $11,60 \mathrm{~nm}$. Full model (1) provided decrease of standard deviation of the weight unit up to $10,96 \mathrm{~nm}$. That is $20 \%$ in general compared to the elementary model (3).

Above all, use of the additional equations (7) helped to calculate all additive degrees of equivalence relative to the single zero of the scale, averaged from all measurements. Due to equations (7) all multiplicative degrees of equivalence are calculated relative to the measurement unit averaged from all measurements.

It is possible to state that mathematically strict processing of such a complicated combination of such a large number of measurements is possible only using the mathematical apparatus ${ }^{[3]}$ and software of the «Metrology Network» type, realizing the general methodology of adjustment by the least square method. Thus, there are no restrictions (except the formal ones) for the quantity of the measurement subjects and objects participating in comparisons, for the quantity of the created in the comparisons process loops and 
quantity of the objects in the loops and quantity of the measurements by subjects in different loops. Formal restrictions are determined by metrological society. Computers capacity used for calculations may be related to formal restrictions as well.

\section{Conclusions}

1. Specially developed «Metrology network» software gives the opportunity to process all complicated combinations of the large quantity of measurement results to receive the comparisons reference values for measurement objects and additive and/or multiplicative degrees of equivalence of measurement subjects (measurement standards).

2. Additive and/or multiplicative degrees of equivalence of the measurement standards are simple for understanding and effective generalized characteristics of the systematic measurement error components by measurement standards, received as comparisons results. They may be used as corrections in the process of calibration or as the reference values for providing further comparisons.

3. Additive degrees of equivalence of measurement standards (interferometers) are proved to be stable and constant characteristic of the systematic measurement error components of the length measurement of steel and ceramic gage blocks during the certain period.

4. «Metrology Network» software realizes the general methodology of adjustment by the least square method. Thus, it removes restrictions on the quantity of measurement subjects and objects participating in comparisons and the quantity of created loops. It also removes restrictions on quantity of possible linking of loops, if under linking of loops may be understood participation of one laboratory (subject) in several loops. Increase of such linking quantity definitely improves general results.

5. Removal of mentioned restrictions opens wide possibilities for logistics optimization of the large quantity of measurement objects (artifacts) circulating among the measurement subjects (measurement standards of the laboratories). Due to such optimization and increase of the measurement objects quantity circulating among the laboratories in the process of comparison it is possible to avoid considerable increase of time on providing comparisons.

6. The main conclusion is that formal restrictions concerning organization of international comprehensive comparisons at any measurement area are withdrawn, where metrological society considers it acceptable.

\section{References}

1. Cox M. G. 2002 The evaluation of key comparison data. Metrologia. 39. pp. 589-595.

https://iopscience.iop.org/article/10.1088/0026-1394/39/6/10

2. Kuzmenko Yu., Samoylenko O. 2018 Processing by least square method of the measurement results for key, regional and supplementary comparison of the measurement standards [Опрацювання методом найменших квадратів результатів вимірювань за ключових, регіональних та додаткових звірень еталонів]. Metrology and Devices. 70, pp. 3-13. [In Ukrainian]

3. Kuzmenko Iu., Samoilenko O. 2021 The measurements results adjustment by the Least Square Method. Measurements infrastructure. 1. https://mi-journal-online.org/index.php/journal/article/ view $/ 1 / 1$

4. Matus M. and etc. 2015 Final report on EURAMET Key Comparison EURAMET.L-K1.2011: Measurement of gauge blocks by interferometry. EURAMET project \# 1218. EURAMET. Vienna, Austria, p. 63.

5. Nazarov N. G. 2002 Metrology. Basic concepts and mathematical models [Метрология. Основные понятия и математические модели]. High School. p.348. [In Russian]

6. Lawson C., Henson R. 1986 Solving Least Squares Problems [Численное решение задач метода наименьших квадратов]. Science. Head Editor phys.-mat. lit., p. 232. [In Russian] 